\title{
Gravitational waves from the Big Bounce
}

\author{
Jakub Mielczarek \\ Marc Kac Complex Systems Research Centre, Jagiellonian University, \\ Reymonta 4, 30-059 Cracow, Poland \\ jakub.f.mielczarek@gmail.com
}

ABSTRACT: In this paper we investigate gravitational waves production during the Big Bounce phase inspired by the Loop Quantum Cosmology. We consider the influence of the holonomy corrections to the equation for tensor modes. We show that they act like additional effective graviton mass, suppressing gravitational waves creation. However, this effects can be treated perturbatively. We investigate the simplified model without these corrections and find its exact analytical solution. For this model we calculate a spectrum of the gravitational waves from the Big Bounce phase. The obtained spectrum decreases to zero for the low energy modes. Based on this observation we indicate that this effect can lead to the low CMB multipoles suppression and gives a potential way to test Loop Quantum Cosmology models. We also consider a scenario with a post-bounce inflationary phase. The obtained power spectrum gives qualitative explanation of the CMB spectra, including low multipoles suppression. This result is a challenge to construct a consistent bounce+inflation model in the Loop Quantum Cosmology. 


\section{Contents}

1. Introduction 1

2. Tensor modes with holonomy corrections 2

3. Toy model 5

3.1 Background dynamics 6

3.2 Mode functions 6

3.3 Power spectrum 9

3.4 Parameter $\Omega_{\mathrm{gw}}$

4. Tensor modes from the pre-bounce phase 13

5. Suppressing low CMB multipoles with a bounce+inflation scenario

6. Summary

\section{Introduction}

Gravitational waves seems to be the best tool to explore early universe. In particular they can be potentially used to verify quantum cosmological models. This idea is based on the fact that gravitational waves produced during the quantum epoch can survive frozen on the super-horizontal scales. Then after re-entering the horizon they can give imprint on the CMB spectrum, which is observed today. From the empirical point of view, the most promising is the B spectrum of the CMB polarization. This spectrum has its source only in the tensor part of perturbation and when observed gives the direct method to investigate relic gravitational waves. It is expected that the PLANCK mission could give an opportunity to detect the B polarization [1]. However, such predictions base on the simple inflationary models.

One of the most promising approaches to quantize gravity is Loop Quantum Gravity [2]. Based on this the theory of the quantum universe, Loop Quantum 
Cosmology [3] arose. This theory predicts that the initial singularity state is replaced by the quantum Big Bounce 四. In this scenario a universe undergoes contraction and then after quantum bounce evolves toward an expanding phase. During the the bounce energy density reach maximal finite energy density $\rho_{c}$. The phenomenological description of the bounce phase can be obtained from the modified Friedmann equation

$$
H^{2}=\frac{\kappa}{3} \rho\left(1-\frac{\rho}{\rho_{\mathrm{c}}}\right) .
$$

where $\kappa=8 \pi G$.

Investigation of the perturbations in the cosmological models are crucial from the point of a large scale structures creation and exploration of the early universe. In the Loop Quantum Cosmology this issue has been studied in Ref. [5, 6, 7, 8]. In the present paper we consider a particular kind of metric perturbations, the gravitational waves. The creation of gravitons in models inspired by the Loop Quantum Cosmology were initially studied in Ref. [9, 10]. Then the equation for the tensor modes with holonomy and inverse volume corrections has been derived in Ref. [11]. Recently the equation with holonomy effects has been applied to the inflationary phase 12 .

In the present paper we investigate the creation of the gravitational waves during the Big Bounce phase. In our considerations we take into account only holonomy effects. The inverse volume corrections exhibit the fiducial cell dependence and are not adequate in the models with the flat background [13]. The production of perturbations in the bouncing cosmologies has been recently studied in the different context in Ref. [14, 15, 16, 17].

\section{Tensor modes with holonomy corrections}

The equation for tensor modes with holonomy corrections has been derived by Bojowald and Hossain [11]. In the source free case these equation takes a form

$$
\frac{d^{2}}{d \tau^{2}} h_{i}+2\left(\frac{\sin 2 \bar{\mu} \gamma \bar{k}}{2 \bar{\mu} \gamma}\right) \frac{d}{d \tau} h_{i}-\nabla^{2} h_{i}+T_{Q} h_{i}=0
$$

where $i=\oplus, \otimes$ indicate polarization state and

$$
T_{Q}=-2\left(\frac{\bar{p}}{\bar{\mu}} \frac{\partial \bar{\mu}}{\partial \bar{p}}\right) \bar{\mu}^{2} \gamma^{2}\left[\frac{\sin (\bar{\mu} \gamma \bar{k})}{\bar{\mu} \gamma}\right]^{4} .
$$


Here $\bar{p}=a^{2}$ and $\bar{k}=\bar{p}^{\prime} / 2 \bar{p}$. The Hamilton equation for the variable $\bar{p}$ takes a form 11]

$$
\bar{p}^{\prime}=2 \bar{p}\left(\frac{\sin 2 \bar{\mu} \gamma \bar{k}}{2 \bar{\mu} \gamma}\right)
$$

what leads to the

$$
\left(\frac{\sin 2 \bar{\mu} \gamma \bar{k}}{2 \bar{\mu} \gamma}\right)=\frac{a^{\prime}}{a} .
$$

The above equality indicates that the friction term in equation (2.1) holds classical form. The effects of holonomies is the additional correction term $T_{Q}$ to the classical equation for tensor modes. This factor acts like an additional effective graviton mass.

To define the correction $T_{Q}$ we need to specify a function $\bar{\mu}$. In general there is some freedom of the choice of this function in the power law form. However, it has been recently shown that for the flat FRW models the only consistent choice is 18

$$
\bar{\mu}=\sqrt{\frac{\Delta}{\bar{p}}}
$$

where $\Delta=2 \sqrt{3} \pi \gamma l_{\mathrm{Pl}}^{2}$, which is called a $\bar{\mu}$-scheme.

Introducing a new variable

$$
u=\frac{a h_{\oplus}}{\sqrt{16 \pi G}}=\frac{a h_{\otimes}}{\sqrt{16 \pi G}}
$$

and performing the Fourier transform

$$
u(\tau, \mathbf{x})=\int \frac{d^{3} \mathbf{k}}{(2 \pi)^{3}} u(\tau, \mathbf{k}) e^{i \mathbf{k} \cdot \mathbf{x}}
$$

we can rewrite the equation (2.1) to the form

$$
\frac{d^{2}}{d \tau^{2}} u(\tau, \mathbf{k})+\left[k^{2}+m_{\mathrm{eff}}^{2}\right] u(\tau, \mathbf{k})=0
$$

where $k^{2}=\mathbf{k} \cdot \mathbf{k}$ and

$$
m_{\mathrm{eff}}^{2}=T_{Q}-\frac{a^{\prime \prime}}{a} .
$$

To calculate this function we must to specify the background dynamics. We consider the model with a free scalar field. In this case evolution of the parameter $\bar{p}$ takes a form [19]

$$
\bar{p}=\left(A+B t^{2}\right)^{1 / 3}
$$


where

$$
A=\frac{1}{6} \kappa \pi_{\phi}^{2} \gamma^{2} \Delta \text { and } B=\frac{3}{2} \kappa \pi_{\phi}^{2} .
$$

Based on definition (2.9) we calculate

$$
m_{\mathrm{eff}}^{2}=\frac{\kappa^{2} \pi_{\phi}^{4}}{4} \frac{\left(t^{2}-\frac{2}{9} \gamma^{2} \Delta\right)}{\left(A+B t^{2}\right)^{5 / 3}} .
$$

In the case $T_{Q}=0$ we obtain

$$
m_{\mathrm{eff}}^{2}\left(T_{Q}=0\right)=\frac{\kappa^{2} \pi_{\phi}^{4}}{4} \frac{\left(t^{2}-\frac{1}{3} \gamma^{2} \Delta\right)}{\left(A+B t^{2}\right)^{5 / 3}} .
$$

In Fig. 1 we show the evolution of the effective masses $m_{\text {eff }}^{2}$ and $m_{\text {eff }}^{2}\left(T_{Q}=0\right)$. Values of these functions are crucial from the point of view of gravitational waves

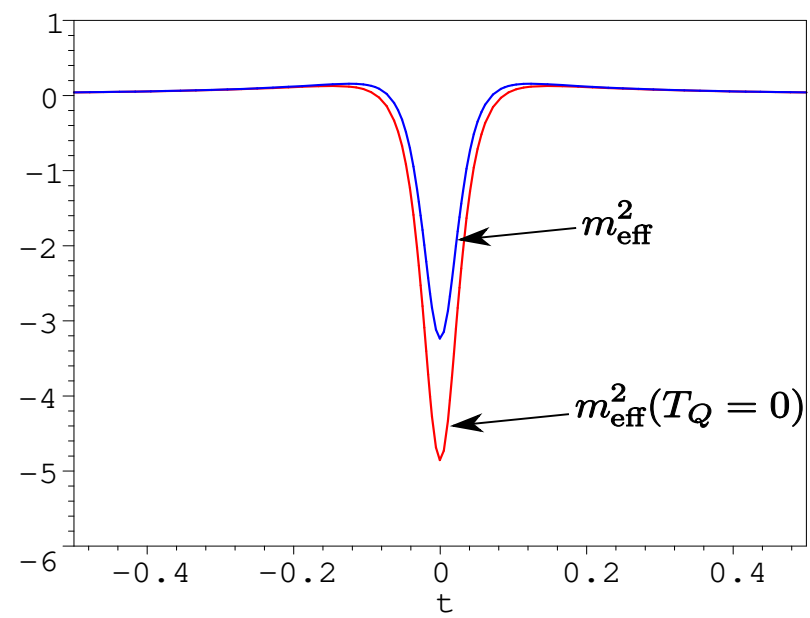

Figure 1: Evolution of the effective masses $m_{\mathrm{eff}}^{2}$ and $m_{\mathrm{eff}}^{2}\left(T_{Q}=0\right)$.

production. Generally, more negative this function is more gravitational waves is produced. We see that the presence of correction $T_{Q}$ leads to suppression of the gravitons production. However, this effect is relatively weak.

For the bouncing universe considered we can write a closed system of equa- 
tions

$$
\begin{aligned}
\frac{d u}{d \tau} & =\pi_{u} \\
\frac{d \pi_{u}}{d \tau} & =-\left[k^{2}+\frac{\kappa^{2} \pi_{\phi}^{4}}{4} \frac{\left(t^{2}-\frac{2}{9} \gamma^{2} \Delta\right)}{\left(A+B t^{2}\right)^{5 / 3}}\right] u, \\
\frac{d t}{d \tau} & =\left(A+B t^{2}\right)^{1 / 6}
\end{aligned}
$$

to describe the evolution of the gravitational waves. This system contains the holonomy correction to the background dynamics as well as to the correction to the equation for tensor modes. Numerical solutions of this system can fully determinate the classical evolution of the gravitational waves during the Big Bounce phase.

In Fig. 2 we show typical solutions for the function $u$ and $h_{i}$ for the vacuum initial condition $u \sim 1 / \sqrt{k}$. It is transparent that the tensor modes are amplified
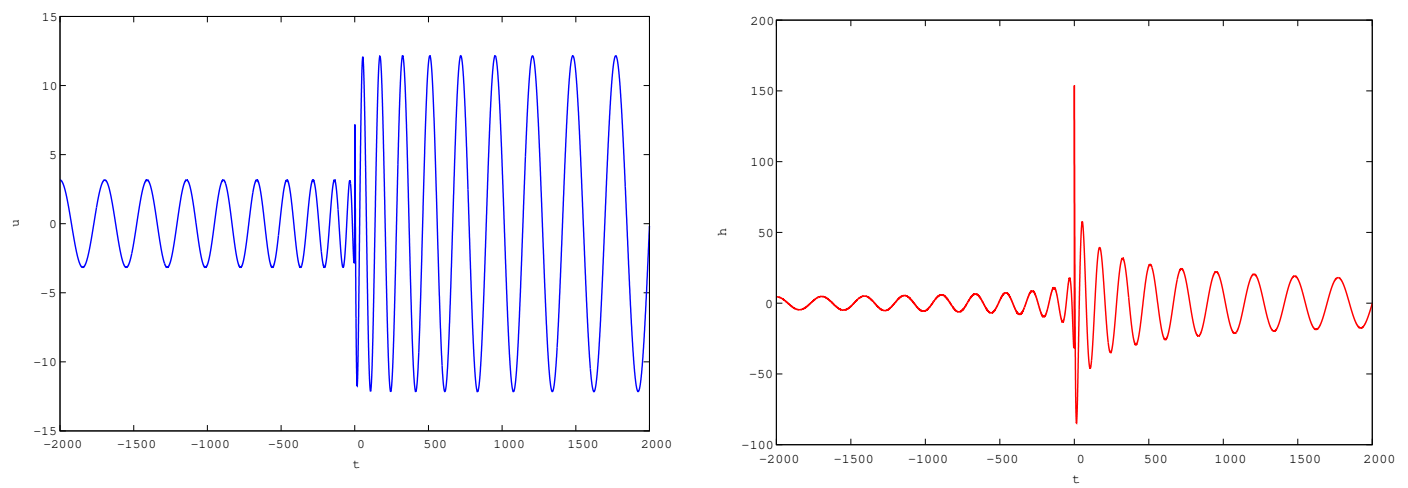

Figure 2: Left : Evolution of the field $u$. Right : Amplification of the tensor modes $h$ during the bounce.

during the bounce phase.

In the next section we aim to determinate qualitative and quantitative properties of the gravitons produced during the bounce phase.

\section{Toy model}

The goal of this section is to give the introduction to the process of particles production during the bounce phase. We show a simple, fully analytical model of the gravitational waves creation during the bounce. The model considered 
does not contain any quantum holonomy corrections to the mode equation. This simplification can be however justified. Namely, as it was shown in the previous section, the holonomy correction $T_{Q}$ leads only to the perturbative modifications.

The model considered is based on the two assumptions:

- we neglect quantum holonomy corrections $T_{Q}$ in the mode equation,

- we assume matter content in the form

$$
\rho=\frac{\rho_{\mathrm{c}}}{a^{2}}
$$

\subsection{Background dynamics}

Plugging condition (3.1) to the modified Friedmann equation (1.1) we find the solution

$$
a(t)=\sqrt{1+\left(t / t_{0}\right)^{2}}
$$

where

$$
t_{0}^{2}=\frac{3}{8 \pi G \rho_{\mathrm{c}}} .
$$

For the further calculations it will be useful to express this solution in terms of the conformal time. Performing transformation $d \tau=d t / a$ we find

$$
a(\tau)=\cosh \left(\tau / t_{0}\right)
$$

\subsection{Mode functions}

Equation (2.8) without the term $T_{Q}$ takes a form

$$
\frac{d^{2}}{d \tau^{2}} u(\tau, \mathbf{k})+\left[k^{2}-\frac{a^{\prime \prime}}{a}\right] u(\tau, \mathbf{k})=0
$$

This equation can be obtained from the action

$$
S_{\mathrm{t}}=\frac{1}{2} \int d \tau d^{3} \mathbf{x}\left[u^{\prime 2}-\delta^{i j} \partial_{i} u \partial_{j} u-m_{\mathrm{eff}}^{2} u^{2}\right]
$$

where

$$
m_{\mathrm{eff}}^{2}=-\frac{a^{\prime \prime}}{a} .
$$

Canonical momenta conjugated to the variable $u$ we obtain from

$$
\pi(\tau, \mathbf{x})=\frac{\delta S_{\mathrm{t}}}{\delta u^{\prime}}=u^{\prime} .
$$


The next step is to quantise this theory. We perform canonical quantisation $(u, \pi) \rightarrow(\hat{u}, \hat{\pi})$ introducing relations of the commutations $[\hat{u}(\mathbf{x}, \tau), \hat{\pi}(\mathbf{y}, \tau)]=$ $i \delta^{(3)}(\mathbf{x}-\mathbf{y})$ and $[\hat{u}(\mathbf{x}, \tau), \hat{u}(\mathbf{y}, \tau)]=[\hat{\pi}(\mathbf{x}, \tau), \hat{\pi}(\mathbf{y}, \tau)]=0$. The operators $\hat{u}, \hat{\pi}$ can be decomposed for the Fourier modes

$$
\begin{aligned}
& \hat{u}(\tau, \mathbf{x})=\frac{1}{2(2 \pi)^{3 / 2}} \int d^{3} \mathbf{k}\left[\hat{u}_{\mathbf{k}}(\tau) e^{i \mathbf{k} \cdot \mathbf{x}}+\hat{u}_{\mathbf{k}}^{\dagger}(\tau) e^{-i \mathbf{k} \cdot \mathbf{x}}\right], \\
& \hat{\pi}(\tau, \mathbf{x})=\frac{1}{2(2 \pi)^{3 / 2}} \int d^{3} \mathbf{k}\left[\hat{\pi}_{\mathbf{k}}(\tau) e^{i \mathbf{k} \cdot \mathbf{x}}+\hat{\pi}_{\mathbf{k}}^{\dagger}(\tau) e^{-i \mathbf{k} \cdot \mathbf{x}}\right] .
\end{aligned}
$$

where

$$
\begin{aligned}
& \hat{u}_{\mathbf{k}}(\tau)=\hat{a}_{\mathbf{k}} f(k, \tau)+\hat{a}_{-\mathbf{k}}^{\dagger} f^{*}(k, \tau), \\
& \hat{\pi}_{\mathbf{k}}(\tau)=\hat{a}_{\mathbf{k}} g(k, \tau)+\hat{a}_{-\mathbf{k}}^{\dagger} g^{*}(k, \tau),
\end{aligned}
$$

and $f(k, \tau)^{\prime}=g(k, \tau)$.

The equation for the mode function takes a form

$$
\frac{d^{2}}{d \tau^{2}} f(k, \tau)+\left[k^{2}+m_{\text {eff }}^{2}\right] f(k, \tau)=0 .
$$

where

$$
m_{\mathrm{eff}}^{2}=-\frac{a^{\prime \prime}}{a}=-\frac{1}{t_{0}^{2}}=-k_{0}^{2} .
$$

With the use of definition

$$
t_{0}^{2}=\frac{3}{8 \pi G \rho_{\mathrm{c}}} \text { and } \rho_{\mathrm{c}}=\frac{\sqrt{3}}{16 \pi^{2} \gamma^{2} l_{\mathrm{Pl}}^{4}} .
$$

and assuming $\gamma=\gamma_{M}=0.12738$ [20 we obtain

$$
k_{0} \simeq \frac{2.38}{l_{\mathrm{Pl}}} .
$$

Now we can find solutions of equation (3.11). We consider two cases $k^{2}>k_{0}^{2}$ and $k^{2}<k_{0}^{2}$.

- Case $k^{2}>k_{0}^{2}$

The solution of equation (3.11) takes a form

$$
f(k, \tau)=A e^{-i \Omega \tau}+B e^{i \Omega \tau}
$$

where $A, B \in \mathbb{C}$ and

$$
\Omega=\sqrt{k^{2}-k_{0}^{2}} .
$$


Performing the normalisation, with the use of the Wronskian condition, we obtain

$$
|A|^{2}-|B|^{2}=\frac{1}{2 \Omega}
$$

We choose advanced modes taking

$$
A=\frac{1}{\sqrt{2 \Omega}} \text { and } B=0
$$

what gives

$$
\begin{aligned}
& f(k, \tau)=\frac{1}{\sqrt{2 \Omega}} e^{-i \Omega \tau}, \\
& g(k, \tau)=f^{\prime}(k, \tau)=-i \sqrt{\frac{\Omega}{2}} e^{-i \Omega \tau} .
\end{aligned}
$$

- Case $k^{2}<k_{0}^{2}$

The solution of equation (3.11) takes a form

$$
f(k, \tau)=A e^{-\bar{\Omega} \tau}+B e^{\bar{\Omega} \tau}
$$

where $A, B \in \mathbb{C}$ and

$$
\bar{\Omega}=\sqrt{k_{0}^{2}-k^{2}}
$$

Performing the normalisation, with the use of the Wronskian condition, we obtain

$$
B A^{*}-A B^{*}=-\frac{i}{2 \bar{\Omega}}
$$

what is fulfilled by

$$
A=\frac{i}{2 \sqrt{\bar{\Omega}}} \text { and } B=\frac{1}{2 \sqrt{\bar{\Omega}}}
$$

In this case we obtain

$$
\begin{aligned}
& f(k, \tau)=\frac{1}{2 \sqrt{\bar{\Omega}}}\left[e^{\bar{\Omega} \tau}+i e^{-\bar{\Omega} \tau}\right] \\
& g(k, \tau)=f^{\prime}(k, \tau)=\frac{\sqrt{\bar{\Omega}}}{2}\left[e^{\bar{\Omega} \tau}-i e^{-\bar{\Omega} \tau}\right] .
\end{aligned}
$$




\subsection{Power spectrum}

The correlation function for the tensor modes takes a form

$$
\begin{aligned}
\left\langle 0\left|\hat{h}_{b}^{a}(\vec{x}, \tau) \hat{h}_{a}^{b}(\vec{y}, \tau)\right| 0\right\rangle & =4 \frac{16 \pi G}{a^{2}} \int \frac{d^{3} k}{(2 \pi)^{3}}|f(k, \tau)|^{2} e^{-i \vec{k} \cdot \vec{r}} \\
& =\int \frac{d k}{k} \mathcal{P}_{T}(k, \tau) \frac{\sin k r}{k r}
\end{aligned}
$$

where we have defined the power spectrum

$$
\mathcal{P}_{T}(k, \tau)=\frac{64 \pi G}{a^{2}} \frac{k^{3}}{2 \pi^{2}}|f(k, \tau)|^{2} .
$$

Our goal now is to determinate this spectrum on the Hubble (horizon) scales. The evolution of the Hubble radius during the bouncing phase considered takes a form

$$
d s^{2}=0 \rightarrow R_{\mathrm{H}}= \pm a(\tau) \int_{0}^{\tau} d \tau^{\prime}= \pm a(\tau) \tau
$$

where + denotes expansion and - denotes contraction. We can also express it in term of the coordinate time $t$, obtaining

$$
R_{\mathrm{H}}= \pm a(t) \int_{0}^{t} \frac{d t^{\prime}}{a\left(t^{\prime}\right)}= \pm t_{0} \sqrt{1+\left(t / t_{0}\right)^{2}} \operatorname{arcsh}\left(t / t_{0}\right) .
$$

This function decreases to zero in the pre-Big Bang phase and increases in the post-Big Bang phase. In Fig. 3 we show evolution of the horizon for the typical bouncing cosmologies. We draw also evolution of the arbitrary length scale $\lambda \propto a$.

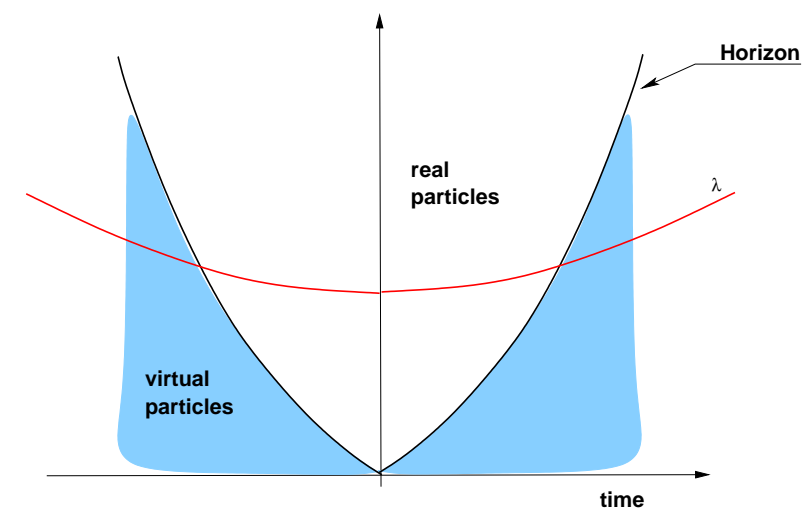

Figure 3: Horizon in the bouncing cosmologies.

We see that during the bouncing phase all length scales finally cross the horizon. 
Quantum fluctuations of the length $\lambda<R_{\mathrm{H}}$ behave like a virtual particles, when fluctuations of the length $\lambda>R_{\mathrm{H}}$ become classical excitations.

The condition $\lambda=R_{\mathrm{H}}$ indicate

$$
k=\frac{2 \pi a}{R_{\mathrm{H}}}=\frac{2 \pi a}{ \pm \tau a}=\frac{2 \pi}{ \pm \tau} .
$$

Plugging it to definition (3.28) and using the mode functions (3.19), (3.25) we determinate the tensor power spectrum on the horizon scales

$$
\mathcal{P}_{T}(k)=\left\{\begin{array}{l}
\frac{16}{\pi}\left(\frac{k_{0}}{m_{\mathrm{Pl}}}\right)^{2}\left(\frac{k}{k_{0}}\right)^{3} \frac{1}{\sqrt{\left(k / k_{0}\right)^{2}-1} \mathrm{ch}^{2}\left(\frac{2 \pi}{k / k_{0}}\right)} \text { for } k>k_{0} \\
\frac{16}{\pi}\left(\frac{k_{0}}{m_{\mathrm{Pl}}}\right)^{2}\left(\frac{k}{k_{0}}\right)^{3} \frac{\mathrm{ch}\left[4 \pi \sqrt{\left(k_{0} / k\right)^{2}-1}\right]}{\sqrt{1-\left(k / k_{0}\right)^{2}} \mathrm{ch}^{2}\left(\frac{2 \pi}{k / k_{0}}\right)} \text { for } k<k_{0}
\end{array}\right.
$$

We plot this spectrum in Fig. 1. We see that for $k<k_{0}$ the spectrum is charac-

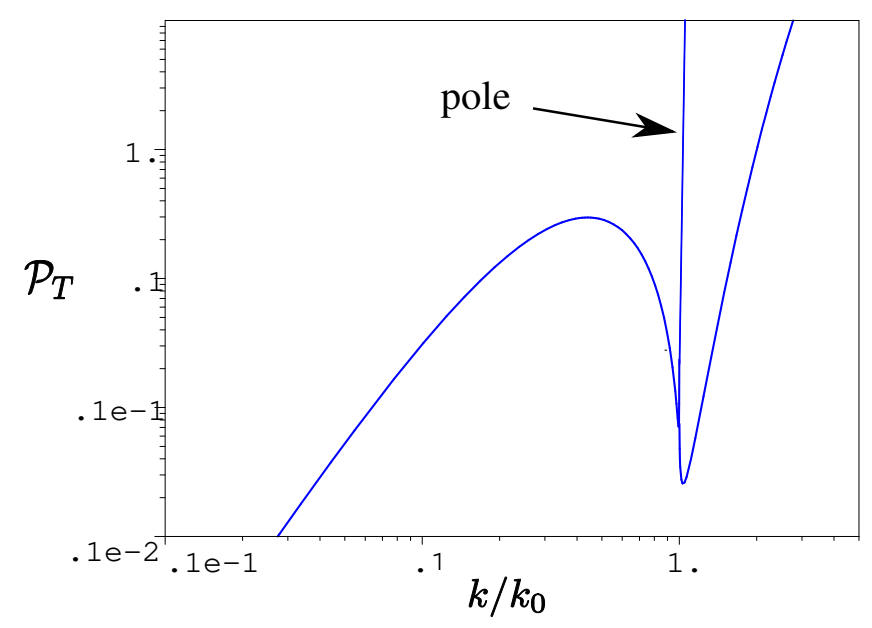

Figure 4: Spectrum of the tensor perturbations on the horizon.

terised by the bump. For $k \rightarrow 0$ the spectrum is decreasing to zero. However, for $k \rightarrow k_{0}$ the spectrum has a pole $\mathcal{P}_{T} \rightarrow \infty$. This pole is however not physical. To show it we consider the correlation function

$$
\left\langle 0\left|\hat{h}_{b}^{a}(\vec{x}, \tau) \hat{h}_{a}^{b}(\vec{x}, \tau)\right| 0\right\rangle=\frac{32 G}{\pi a^{2}} \int_{0}^{\infty} d k k^{2}|f(k, \tau)|^{2},
$$

where

$$
\begin{aligned}
|f(k, \tau)|^{2} & =\Theta\left(k-k_{0}\right) \frac{1}{2 \sqrt{k^{2}-k_{0}^{2}}} \\
& +\Theta\left(k_{0}-k\right) \frac{1}{2 \sqrt{k_{0}^{2}-k^{2}}} \mathrm{ch}\left[2 \sqrt{k_{0}^{2}-k^{2} \tau}\right] .
\end{aligned}
$$


The correlation function is a physical quantity and can indicate which features of the spectrum $\mathcal{P}_{T}$ are real divergences. As we can see, near the pole $k=k_{*}$ the correlation function is determined by the integrals

$$
I_{1}=\int d k \frac{k^{2}}{\sqrt{k^{2}-k_{0}^{2}}}=\left\{x=\frac{k}{k_{0}}\right\}=\frac{k_{0}^{2}}{2}\left[x \sqrt{x^{2}-1}-\ln \left|x+\sqrt{x^{2}-1}\right|\right]
$$

and

$$
I_{2}=\int d k \frac{k^{2}}{\sqrt{k_{0}^{2}-k^{2}}}=\left\{x=\frac{k}{k_{0}}\right\}=\frac{k_{0}^{2}}{2}\left[-x \sqrt{1-x^{2}}-\arcsin x\right] .
$$

Both of them are finite for the $x=1$ and give a finite contribution to the definition of the correlation function.

For $k \rightarrow \infty$ the power spectrum exhibit a UV divergence. In fact, the region $k>k_{0}$ traces the Planck scales and a different approach should be used to determinate properties of the quantum fluctuations. Another important issue is to indicate which of the modes survive frozen above the Hubble radius. In fact, not all of them but only these with $k<k_{0}$. It can be seen from the asymptotic solutions of equation (3.5). Namely, in the regime $k^{2} \ll\left|a^{\prime \prime} / a\right|=k_{0}^{2}$ we have solution

$$
h_{i} \simeq A_{k}+B_{k} \int^{\tau} \frac{d x}{a^{2}(x)} .
$$

Here we have a constant contribution $A_{k}$ which freezes the amplitude of the gravitational waves. In this regime the spectrum does not change during the evolution above the horizon and will be the same when re-enters the horizon. In the regime $k^{2} \gg\left|a^{\prime \prime} / a\right|=k_{0}^{2}$ we have decaying solutions

$$
h_{i} \simeq \frac{e^{ \pm i k \tau}}{a}
$$

These modes does not lead to the classical fluctuations when re-enter horizon.

Summing up, the spectrum $\mathcal{P}_{T}$ on the second branch of the horizon is free form the UV divergent part for the $k>k_{0}$. The only contribution to this spectrum comes from the bump for the $k<k_{0}$.

\subsection{Parameter $\Omega_{\mathrm{gw}}$}

To describe the spectrum of gravitons it is common to use the parameter

$$
\Omega_{\mathrm{gw}}(\nu)=\frac{\nu}{\rho_{*}} \frac{d \rho_{\mathrm{gw}}}{d \nu}
$$


where $\rho_{\mathrm{gw}}$ is the energy density of gravitational waves and $\rho_{*}$ is present critical energy density. Our goal in this section is to calculate the function $\Omega_{\mathrm{gw}}(\nu)$ for the gravitons produced during the Big Bounce phase.

We consider the creation of the gravitons during the transition from some initial to final states. The initial vacuum state $\left|0_{\text {in }}\right\rangle$ is determined by $\hat{a}_{\mathrm{k}}\left|0_{\text {in }}\right\rangle=0$, where $\hat{a}_{\mathrm{k}}$ is the initial annihilation operator for $\tau_{i}$. The relation between annihilation and creation operators for the initial and final states is given by the Bogoliubov transformation

$$
\begin{aligned}
& \hat{b}_{\mathbf{k}}=B_{+}(k) \hat{a}_{\mathbf{k}}+B_{-}(k)^{*} \hat{a}_{-\mathbf{k}}^{\dagger}, \\
& \hat{b}_{\mathbf{k}}^{\dagger}=B_{+}(k)^{*} \hat{a}_{\mathbf{k}}^{\dagger}+B_{-}(k) \hat{a}_{-\mathbf{k}}
\end{aligned}
$$

where $\left|B_{+}\right|^{2}-\left|B_{-}\right|^{2}=1$. Because we are working in the Heisenberg description the vacuum state does not change during the evolution. It results that $\hat{b}_{\mathbf{k}}\left|0_{\text {in }}\right\rangle=$ $B_{-}(k)^{*} \hat{a}_{-\mathbf{k}}^{\dagger}\left|0_{\text {in }}\right\rangle$ is different from zero when $B_{-}(k)^{*}$ is a nonzero function. This means that in the final state graviton field considered is no more in the vacuum state without particles. The number of produced particles in the final state is given by

$$
\bar{n}_{\mathbf{k}}=\frac{1}{2}\left\langle 0_{\text {in }}\left|\left[\hat{b}_{\mathbf{k}}^{\dagger} \hat{b}_{\mathbf{k}}+\hat{b}_{-\mathbf{k}}^{\dagger} \hat{b}_{-\mathbf{k}}\right]\right| 0_{\text {in }}\right\rangle=\left|B_{-}(k)\right|^{2} .
$$

The energy density of gravitons is given by

$$
d \rho_{\mathrm{gw}}=2 \cdot \hbar \omega \cdot \frac{4 \pi \omega^{2} d \omega}{(2 \pi c)^{3}} \cdot\left|B_{-}(k)\right|^{2} .
$$

where we used definition (3.41). The expression for the parameter $\Omega_{\mathrm{gw}}$ defined by (3.38) takes now the form

$$
\Omega_{\mathrm{gw}}(\nu)=\left\{\begin{array}{cl}
0 & \text { for } k>k_{0} \\
\Omega_{0} \cdot \nu^{4} \cdot \sinh ^{2}\left[\sqrt{1-\left(k / k_{0}\right)^{2}}\left(\tau_{\mathrm{i}}-\tau_{\mathrm{f}}\right) k_{0}\right] & \text { for } k \leq k_{0}
\end{array}\right.
$$

where

$$
k=2 \pi \nu \frac{a_{0}}{a_{\mathrm{f}}}
$$

and

$$
\Omega_{0}=\frac{\hbar c}{c^{4}} \frac{16 \pi^{2}}{\rho_{*}}=3.66 \cdot h_{0}^{-2} \cdot 10^{-49}\left[\mathrm{~Hz}^{-4}\right] .
$$

For the model with the inflationary phase we have $\frac{a_{0}}{a_{\mathrm{f}}} \simeq 10^{56}$. Another value which must be specified is the duration of the bounce. We assume that $\tau_{\mathrm{i}}=-20 l_{\mathrm{Pl}}$ and the $\tau_{\mathrm{f}}=20 l_{\mathrm{Pl}}$. 


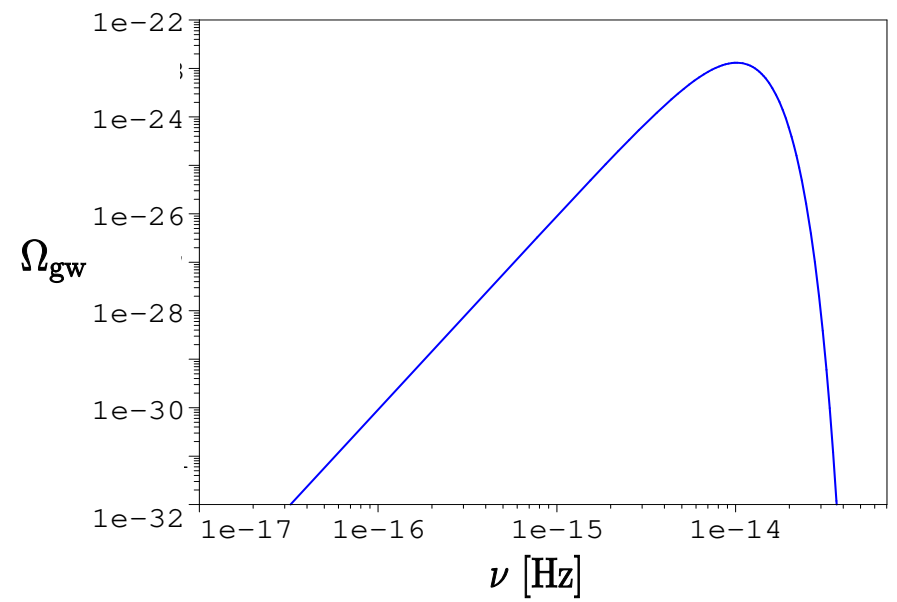

Figure 5: Function $\Omega_{\mathrm{gw}}(\nu)$ for the $\tau_{\mathrm{i}}=-20 l_{\mathrm{Pl}}$ and the $\tau_{\mathrm{f}}=20 l_{\mathrm{Pl}}$.

In Fig. 5 we show the function $\Omega_{\mathrm{gw}}(\nu)$ for the setup considered. The spectrum is characterised by the bump with the maximal frequency

$$
\nu_{\max } \simeq 7 \cdot 10^{-14}[\mathrm{~Hz}] .
$$

The presented result approves our previous consideration. Namely, there is no production of the gravitons with frequencies above some $\nu_{\max }$. The only contribution to the spectrum comes from the bump.

\section{Tensor modes from the pre-bounce phase}

The bouncing solution (2.10) in the limit $t \rightarrow \pm \infty$ gives

$$
a(t) \propto|t|^{1 / 3} \propto|\tau|^{1 / 2} .
$$

In this regime the correction $T_{Q}$ vanishes and the expression for the effective mass simplifies to

$$
m_{\mathrm{eff}}^{2}=-\frac{a^{\prime \prime}}{a}=\frac{1}{4} \frac{1}{\tau^{2}} .
$$

The equation for the mode functions takes a form

$$
\frac{d^{2}}{d \tau^{2}} f(k, \tau)+\left[k^{2}+\frac{1}{4} \frac{1}{\tau^{2}}\right] f(k, \tau)=0,
$$

The normalised solution of this equation has a form

$$
f(k, \tau)=\frac{\mathcal{N}}{\sqrt{2 k}} \sqrt{-\tau k} H_{0}^{(1)}(-\tau k)
$$


where

$$
\mathcal{N}=\sqrt{\frac{\pi}{2}} e^{i \pi / 4}
$$

With the use of definitions (3.28) and (4.4) we obtain the power spectrum for the pre-bounce phase in the form

$$
\mathcal{P}_{T}(k)=\sqrt{\frac{12}{\pi}}\left|H_{0}^{(1)}(2 \pi)\right|^{2}\left(\frac{k}{k_{\#}}\right)^{3} \text { for } k \rightarrow 0,
$$

where $k_{\#}$ is some constant.

It is important to note that this part of the spectrum does not depend on the quantum gravitational effects. It is in opposite to the predictions from the inflationary models, where low energy modes come from a high energy region. Here, low energy modes are produced in the low energy pre-Big Bang state. So, the present largest scale structures have their origin in the semi-classical pre-Big Bang phase rather than in the deep quantum regime.

\section{Suppressing low CMB multipoles with a bounce+inflation scenario}

In this section we consider scenario with an inflationary phase taking place after the bounce. In Fig. 6 we show the resulting evolution of the horizon. We see that

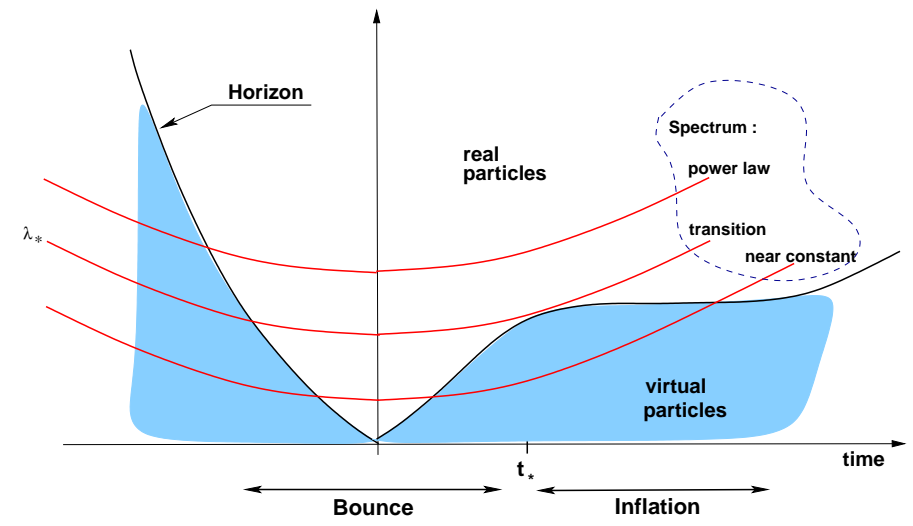

Figure 6: Evolution of the horizon in the bounce+inflation model.

the horizon is firstly crossed by the modes of the length $\lambda>\lambda_{*}$. These modes lives frozen on the super-horizontal scales and re-enter the horizon in the post-Big Bang phase. Additionally they are not sensitive for the quantum gravitational 
effects close to the bounce. As it was shown in the previous section they leads to the power spectrum in the form

$$
\mathcal{P}_{T}(k) \propto k^{3} \text { for } k \ll k_{*} \text {. }
$$

These fluctuations can lead directly to the CMB fluctuations on the large angular scales. Modes of the length $\lambda<\lambda_{*}$ also cross the horizon in the pre-Big Bang phase but re-enter shortly after bounce. They decay shortly after this and become a quantum fluctuations. Then, during the inflationary phase they cross the horizon again. The spectrum of these fluctuations will be nearly flat as predicted by the inflationary models

$$
\mathcal{P}_{T}(k)=\mathcal{A} \text { for } k \gg k_{*} \text {. }
$$

The loop holonomy corrections to the inflationary spectrum were studied in Ref. 21, 22.

Finally, we should obtain the spectrum of the tensor perturbations in the form shown in Fig. 7 .

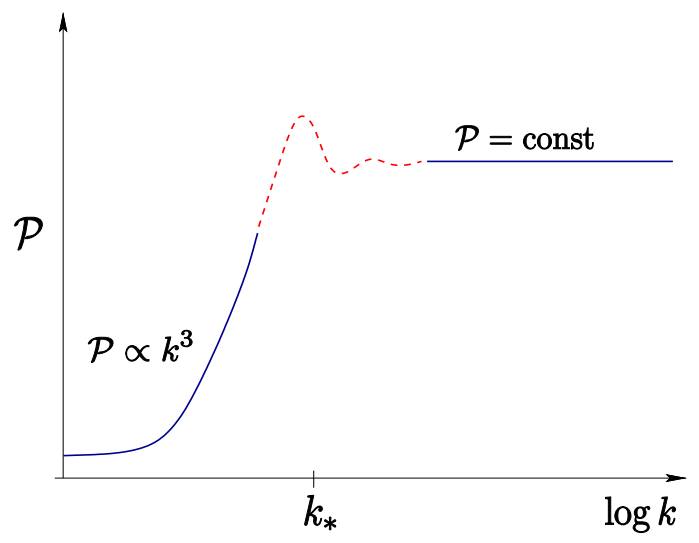

Figure 7: The schematic picture of the power spectrum expected for the bounce+inflation model.

To the describe power spectrum in a continuous way we propose the phenomenological formula

$$
\mathcal{P}(k)=\mathcal{A}\left(\frac{k}{k_{*}}\right)^{3} \frac{1}{1+\left(\frac{k}{k_{*}}\right)^{3}},
$$

which interpolates the two regimes considered. We plot this function in Fig. 8. 


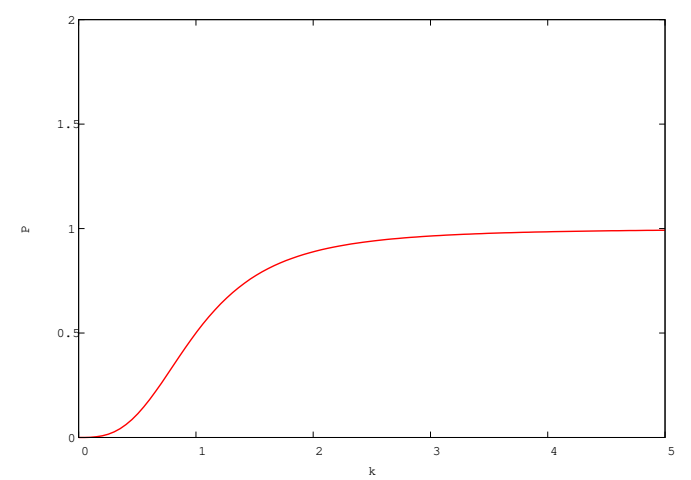

Figure 8: Phenomenological spectrum for the bounce+inflation model with $\mathcal{A}=1$ and $k_{*}=1$.

The spectrum in the same form should be also obtained for the scalar modes of fluctuation. In this context similar models were studied in Ref. [23, 24]. They have shown that a transitional regime in the spectrum can have form of the oscillations, as it was shown in Fig. 7. However, in the cited papers evolution was obtained form the specific dynamics of the single inflationary field. Namely, no quantum gravitational corrections was used to obtain the bounce phase.

An interesting property of the obtained spectra is damping of the low multipoles in the CMB spectra. Such an effect is in fact observed [25]. This gives potentially the possibility to test bouncing cosmologies. If the bounce is present we should observe low multipoles suppression. However, this effect can have also other origins and on the present stage of observations it is not possible to indicate that it is truly the remnant of the Big Bounce.

The important feature of the presented scenario is that none of the primordial perturbations produced in the deep quantum gravitational regime gives seeds to the structures formation. All perturbations come from either the pre-Big Bang semi-classical phase or from the post-Big Bang inflationary phase. It is therefore hard to investigate observationally the deep quantum regime. However, we can potentially observe the classical pre-Big Bounce branch which is the result of the quantum gravitational effects.

\section{Summary}

In this paper we have considered the gravitational waves creation during the Big Bounce phase inspired by the Loop Quantum Cosmology. We have studied effects of the holonomy corrections to the equation for tensor modes. We have 
shown that they lead to the suppression of the gravitons production. However, this effects is not dominant and can be treated perturbatively. In fact, to obtain qualitative results it is justified to neglect this contribution. Based on the above studies we have solved the simplified model of the gravitons production during the bounce phase. We have derived values the power spectrum $\mathcal{P}_{T}$ and the parameter $\Omega_{\mathrm{gw}}$. The obtained spectrum for the bounce has a form of the bump. It decreases to zero for the energies tending to zero and for some high energy scale. This is a typical property of the bouncing cosmologies. The similar behaviour is also expected for the scalar perturbations. The perturbations form the pre-Big Bang phase gives a direct imprint for the large scale structures. In particular they can lead to the suppression of the low CMB multipoles. However, the bounce phase itself does not lead to observed features of the CMB spectra. The post-bounce inflationary phase is required. Such a model with the bounce and the following inflationary phase is not yet constructed in the Loop Quantum Cosmology. It is therefore a challenge to construct it in a consistent way.

\section{Acknowledgments}

I am grateful to prof. Marek Szydłowski and dr Adam Krawiec for careful reading the manuscript. This work has been supported by the Marie Curie Host Fellowships for the Transfer of Knowledge project COCOS (Contract No. MTKD-CT2004-517186).

\section{References}

[1] [Planck Collaboration], arXiv:astro-ph/0604069.

[2] A. Ashtekar and J. Lewandowski, Class. Quant. Grav. 21 (2004) R53 [arXiv:gr-qc/0404018].

[3] M. Bojowald, Living Rev. Rel. 8 (2005) 11 [arXiv:gr-qc/0601085].

[4] A. Ashtekar, T. Pawlowski and P. Singh, Phys. Rev. Lett. 96 (2006) 141301 [arXiv:gr-qc/0602086].

[5] M. Bojowald, H. H. Hernandez, M. Kagan, P. Singh and A. Skirzewski, Phys. Rev. D 74 (2006) 123512 [arXiv:gr-qc/0609057].

[6] M. Bojowald, H. Hernandez, M. Kagan, P. Singh and A. Skirzewski, Phys. Rev. Lett. 98 (2007) 031301 [arXiv:astro-ph/0611685]. 
[7] M. Bojowald, AIP Conf. Proc. 917 (2007) 130 [arXiv:gr-qc/0701142].

[8] M. Bojowald, G. M. Hossain, M. Kagan and S. Shankaranarayanan, arXiv:0806.3929 [gr-qc].

[9] J. Mielczarek and M. Szydlowski, Phys. Lett. B 657 (2007) 20 [arXiv:0705.4449 [gr-qc]].

[10] J. Mielczarek and M. Szydlowski, arXiv:0710.2742 [gr-qc].

[11] M. Bojowald and G. M. Hossain, Phys. Rev. D 77 (2008) 023508 [arXiv:0709.2365 [gr-qc]].

[12] A. Barrau and J. Grain, arXiv:0805.0356 [gr-qc].

[13] A. Ashtekar, T. Pawlowski and P. Singh, Phys. Rev. D 74 (2006) 084003 [arXiv:gr-qc/0607039].

[14] M. Gasperini, M. Giovannini and G. Veneziano, Phys. Lett. B 569 (2003) 113 [arXiv:hep-th/0306113].

[15] P. Creminelli and L. Senatore, JCAP 0711 (2007) 010 [arXiv:hep-th/0702165].

[16] Y. F. Cai, T. Qiu, R. Brandenberger, Y. S. Piao and X. Zhang, JCAP 0803 (2008) 013 [arXiv:0711.2187 [hep-th]].

[17] M. Novello and S. E. P. Bergliaffa, Phys. Rept. 463 (2008) 127 [arXiv:0802.1634 [astro-ph]].

[18] A. Corichi and P. Singh, arXiv:0805.0136 [gr-qc].

[19] J. Mielczarek, T. Stachowiak and M. Szydlowski, Phys. Rev. D 77 (2008) 123506 [arXiv:0801.0502 [gr-qc]].

[20] K. A. Meissner, Class. Quant. Grav. 21 (2004) 5245 [arXiv:gr-qc/0407052].

[21] X. Zhang and Y. Ling, JCAP 0708 (2007) 012 [arXiv:0705.2656 [gr-qc]].

[22] M. Artymowski, Z. Lalak and L. Szulc, arXiv:0807.0160 [gr-qc].

[23] C. R. Contaldi, M. Peloso, L. Kofman and A. Linde, JCAP 0307 (2003) 002 [arXiv:astro-ph/0303636].

[24] Y. S. Piao, B. Feng and X. m. Zhang, Phys. Rev. D 69 (2004) 103520 [arXiv:hep-th/0310206].

[25] A. Shafieloo and T. Souradeep, Phys. Rev. D 70 (2004) 043523 [arXiv:astro-ph/0312174]. 